\title{
Mesenchymal Stem Cells Derived Extracellular Vesicles
}

\section{Lamiaa Ibrahim Abd ElFattah}

\author{
Review \\ Department of Medical Histology and Cell Biology, Faculty of Medicine, Cairo University, \\ Article \\ Cairo, Egypt
}

\begin{abstract}
Mesenchymal stem cells (MSCs) since their initial discovery have been a subject of intense investigations. Paracrine activity is the predominent mechanism of MSCs in tissue repair via the release of extracellular vesicles (EVs). Mesenchymal stem cells derived extracellular vesicles (MSCs-EVS) are small spherical membrane bounded fragments in the conditioned medium of MSCs. EVs differ in their origin, size and contents,yet they have the ability to induce changes in the recipient cells. Several studies were carried out investigating the therapeutic potentials of MSCs-EVs in many fields including cardiovascular, respiratory, renal diseases and cancer. They were also reported to have immunomodulatory effects. EVs have many advantages over the use of MSCs including the ability to home to target tissue with no organ accumulation, no toxicity and no long term maldifferentiation of engrafted cells. In addition, EVs are able to deliver biological materials safer than current methods including viral vehicles. MSCs-EVs are promising biomedical tools in stem cell based therapies.
\end{abstract}

Keywords: Extracellular vesicles, cell based therapy, stem cells

Revised: 7 June 2017, Accepted: 14 June 2017

Corresponding Author: Lamiaa Ibrahim AbdElFattah, Tel.: 00201222741975, E-mail: lamiaafattah@ymail.com

ISSN: 2536-9172, June 2017, Vol. 1, No. 1

\section{BACKGROUND}

Mesenchymal stem or stromal cells (MSCs), since their initial discovery by Alexander Friedenstein in 1960s, have been a subject of intense investigations ${ }^{[1]}$. MSCs based therapy is a great alternative to current therapeutic approaches in regenerative medicine due to their ability to self-renewal and differentiation into various tissues ${ }^{[2]}$. A paracrine activity of MSCs in tissue repair have been suggested as a predominant mechanism. This occurs via exocytosis of a secretome containing both extracellular vesicles and released factors; including growth factors and cytokines $^{[3]}$. This provides a microenviroment that induce angiogenesis, support regeneration and reduce further tissue injury ${ }^{[4]}$. MSCs derived extracellular vesicles EVs appear to be a very important tool to get the benefits of MSC therapy by using cell-free strategies depending on MSC secretome, thus reducing the associated risks of using $\mathrm{MSC}^{[5]}$.

\section{$M S C$ - derived EVS}

Extracellular vesicles EVs are small, spherical membrane bounded fragments contained in the conditioned medium of MSCs. Interestingly, they are capable of inducing changes in the recipient cells ${ }^{[6]}$. EVs are secreted by a wide range of cells including mast cells, platelets ,dendritic cells, T and B lymphocytes, as well as, MSCs either bone marrow, placenta or adipose derived. In vivo, they can be isolated from body fluids such as serum, urine and broncho alveolar lavage fluid ${ }^{[7]}$. EVs were reported to adhere to extracellular matrix forming reservoir which could be released on need as in case of inflammation and damage ${ }^{[8]}$.

\section{Types and Characteristics of $E V S$}

\section{Types}

The EVs include exosomes, microvesicles and apoptotic bodies. They differ according to size, origin and contents. Moreover, the number and nature is affected by age, gender, fasting, circadian rhythm, physical activity and medication ${ }^{[9]}$. Exosomes range between $50-150 \mathrm{~nm}$ in size originating from multivesicular bodies MVBs. This occurs by inward budding of the multivesicular body (MVB) limiting membrane forming intraluminal vesicle (Fig. 1) which fuse with the plasma membrane to be released as exosome in a way which is not dependent on calcium influx ${ }^{[10]}$. In contrast, the microvesicles range in size from $150-1000 \mathrm{~nm}$, arise from plasma membrane by budding of cytoplasmic protrusions (Fig.1) in a process dependent on calcium ions which are responsible for asymmetric distribution of plasma membrane 
phospholipids yielding the formation of the microvesicles ${ }^{[7]}$. Finally, a large type of EVs more than $1 \mathrm{um}$ is the apoptotic body, which is a derivative of dying cells $^{[11]}$.

\section{Content}

Regarding the contents of EVs, exosomes contain proteins and lipids, DNA, mRNA and microRNA (miRNA). Additionally, microvesicles reported to have cell organelles ${ }^{[10]}$. Apoptotic bodies contain DNA, non codingRNAs as well as cell organelles ${ }^{[1]}$. MSC-derived EVs are rich in classes of RNA which upon transfer to target cells, got translated into proteins. The transcripts are involved in the control of transcription, cell proliferation and immune regulation ${ }^{[12]}$. The RNA profile of exosomes derived from different sources was compared. Interestingly, bone marrow-derived MSCs (BM-MSCs) and adiposederived MSCs (ASCs) share similar representatives of RNAs despite differences in the relative proportions ${ }^{[7]}$. Micro RNA (miRNA) is a single strand of RNA with about 22 nucleotide length, single miRNA could repress several genes ${ }^{[13]}$. Both non coding RNA and miRNAs of EVs control gene expression post-transcriptionally, so they are related to cell survival and differentiation as well as regulation of the immune system ${ }^{[7]}$.

\section{Surface markers}

MSC-derived EVs are characterized by surface molecules (Table 1) that are related to their cell of origin, such as CD44, CD29, CD105 and CD73. Among the EVs, exosomes are best characterized as they conserve sets of proteins including tetraspanins, for cell targeting (CD9,CD63 and CD81) that are considered unique markers for exosomes. Other markers include, heat shock proteins (Hsp60, Hsp70 and Hsp90), ALG2- interacting protein X (Alix), tumour susceptibility gene (Tsg101), Major histocompatibility complex I and II , integrins and annexins. Markers of microvesicles involve integrins, flotillins and tetraspanins, while apoptotic bodies has surface markers of macrophages ${ }^{[7]}$.

Analysis of MSC sexosomes identified 857 proteins that are involved in a wide array of cellular processes. It was identified that miR-19a is cardioprotective, miR22 improve ischaemic injury and reduce apoptosis and 20S proteasome promote angiogenesis and inhibit oxidative stress ${ }^{[14]}$. Interestingly, it was found that the exchange of mRNA, miRNA and proteins may be bidirectional, MVs released from injured cells may reprogram the phenotype of stem cells to induce differentiation. Conversely, MVs released from stem cells may reprogram tissue-injured cells with activation of regenerative programs that include cell dedifferentiation, cell cycle re-entry and redifferentiation ${ }^{[15]}$.

\section{Mechanism of action of EVs}

EVs play a pivotal role in intercellular communication with ability to modify the target cell activity via different mechanisms involving exchange of information by direct interaction with surface receptor, receptor transfer between cells, protein transfer or transfer of genetic information via mRNA and miRNA to target cell ${ }^{[16,17]}$. Moreover, the ways by which EVs enter the target cell could be fusion with cell membrane, endocytosis or receptor mediated binding ${ }^{[18]}$.

\section{Isolation of $E V s$ and method of preparation}

In vivo, EVs can be isolated from different body fluids. In vitro, EVs are obtained from cultured media of cells grown in absence of serum by standardized specific protocols characterized by ultracentrifugation, ultrafiltration and immunoprecipitation using magnetic cell beads loaded with antibody ${ }^{[19]}$

Briefly, to obtain EVs, MSCs has to be cultured in DMEM without fetal bovine serum (FBS) and with added $0.5 \%$ bovine serum albumin (BSA) overnight. Then collect the conditioned medium and store at $-80^{\circ}$, centrifuge the medium at 2,000 $\mathrm{g}$ for 20 minutes in order to remove debris followed by ultracentrifugation at 100,000 $\mathrm{g}$ for one hour at $4^{\circ}$. Washing the vesicles, serum free, then submission for the second ultracentrifugation under same conditions. The vesicles to be stored at $-80^{\circ}$ for use. Interestingly, it was recorded that 5 X 105 MSCs release about 100ug microvesicles overnight ${ }^{[20]}$.

\section{How to detect or visualize EVs?}

Since the size of exosomes is smaller than that to be distinguished by flow cytometry, other methods were used. Antibody based exosomal identification is a method in which antibodies against surface markers including tetraspanins, Alix and Tsg101 could be used. Exosomes can be bounded to latex beads which bind to antibodyconjugated beads followed by staining of the bounded exosomes $^{[13]}$. Exosomes can be clearly seen by transmission electron microscopy using the purified exosomal suspension to be placed in the grid, followed by staining with uranyl acetate and methylcellulose. Exosomes appear as double membrane vesicles with cup shaped characteristic that represents an artefact of drying during preparation which is a constant finding for exosomes ${ }^{[21]}$. Moreover, confirmation of the presence of exosomes within a preparation could be achieved by staining against antigens mentioned before, followed by secondary staining using gold nanoparticles which could be clearly seen on electron micrographs ${ }^{[13]}$. Another technology is using nanoparticle tracking analysis NTA, which is a light microscopy mounted by high definition video camera. The sample passed through a flow cell on the stage of the microscope at a constant rate with a laser beam shine through the flow 
cell allowing the camera to record. Then the NTA software calculate the size of particles using a special equation ${ }^{[22]}$. Alternatives include, dynamic light scattering (DLS) in which the laser beam pass through the sample followed by calculation of the speed of particles. Moreover, using the dye PKH67 allow visualization of exosomes taken up into the target cell by fluorescent microscopy. Other methods to detect exosomesinclude, the use of atomic force microscopy and micro nuclear magnetic resonance ${ }^{[13]}$.

\section{Is it Possible to modulate EVs release?}

Enhancement of the beneficial effects of EVs is of great interest in the field of tissue repair. Exposure to hypoxia and coculture with human or animal serum derived from pathological conditions was found to induce the release and augment the effects of MSC-derived EVs due to the presence of cytokines and growth factors that provide the needed microenviroment. In contrast, pretreatment of EVs with RNase was found to inhibit the biological effects ${ }^{[7]}$. Interestingly, pre-treatment of MSCderived EVs with specific growth factors could change the phenotype and content of the vesicles. It was reported that treatment of adult stem cells (ASCs) with platelet derived growth factor resulted in enhanced angiogenic activity of the derived $\mathrm{EVs}^{[23]}$. Moreover, the efficiency of uptake of EVs by the target cells was observed to be directly related to acidity, both intracellular and microenviromental ${ }^{[24]}$.

\section{Therapeutic potentials of EVs}

Several studies were carried out to clarify the beneficial effects of MSC-derived EVs in many diseases including respiratory, cardiovascular and renal diseases ${ }^{[4,7,20]}$.

\section{A-Respiratory Diseases}

Regarding asthma, administration of MSC-derived EVs was more effective than MSCs themselves in reducing lung inflammation and allergic hyper-responsiveness. Moreover, blocking the release of EVs revealed absence of the protective effects ${ }^{[25]}$. In cases of hypoxia induced pulmonary arterial hypertension, EVs was found to protect against the right ventricular systolic pressure elevation and consequently, protected against right ventricular hypertrophy. Additionally, the use of EVs depleted media had no effect ${ }^{[26]}$. Acute respiratory distress syndrome (ARDS) is a clinical condition with progressive respiratory failure and mortality rate about $40 \%$. EVs was reported to reduce lung inflammation in case of endotoxin induced ARDS $^{[27]}$. In addition, MSCs-derived EVs were found to have antimicrobial effect that reduce bacterial growth and improve in cases of pneumonia ${ }^{[28]}$.

\section{B-Cardiovascular Diseases}

Cardiac tissue repair and remodelling was found to be associated with MSCs-derived factors, they were able to preserve the contractile capacity of myocardium, inhibit cardiomyocytes apoptosis and contribute to neoangiogenesis. Moreover, they were shown to limit the size of infarction and improve the systolic function and echocardiographic parameters ${ }^{[4]}$.

\section{C-Renal Diseases}

MSCs-derived microvesicles were shown to have beneficial effects during recovery of tissue and organ injury including kidney, where they contribute to the restoration of damaged kidney by reducing apoptosis and protecting against fibrosis ${ }^{[29]}$.

\section{D- Immunomodulation}

MSCs-derivedEVswerereportedasimmunomodulatory therapeutic agents, based on their ability to stimulate secretion of anti-inflammatory cytokines and generation of regulatory $\mathrm{T}$ cells ${ }^{[30]}$. Exosomes containing miR$181 \mathrm{c}$ played an important role in attenuating burn induced inflammation ${ }^{[31]}$. Moreover, MSCs were proposed for organ transplantation. MSCs derived EVs suppress allograft rejection in a rat model of renal transplantation ${ }^{[8]}$.

\section{E-Cancer}

Despite the data indicating that MSCs are incorporated into the microenviroment of tumours and stimulate their growth $^{[32]}$. A new hope was addressedby the findings of Lou and his colleagues ${ }^{[33]}$, they found that miR-122 loaded exosomes promote chemosensitivity of hepatocellular carcinoma (HCC) cells. Thus, arising a promising strategy for chemotherapy, via vehicle mediated delivery of EVs. Interestingly, in case of cancer breast, transfer of exosomes containing miR- $127,-197,-222$ was shown to reduce the growth of cultured breast cancer cells ${ }^{[1]}$.

\section{MSC secretome or EVs?}

Since the first analysis of BM-MSCs secretory products was held at 2003, MSCs conditioned media (MSCs-CM) have been a subject of intense investigations for analysis of MSCs secretome. Studies were performed in vitro via collection of conditioned media by cells for $12-48 \mathrm{~h}$ of culture. Secretome profiling revealed the presence of both EVs and released factors, including ECM proteins, factors promoting vasculo-genesis and endothelial tube formation and others promoting MSC survival and self-renewal. The conditioned media (CM) most abundant factor is pigment epithelium derived factor (PEDF) which is a predominant fibroblast chemoattractant ${ }^{[4]}$.

\section{Potential uses of EVs over MSCs}

EVs are important vehicles for drug delivery that can carry both hydrophilic and lipophilic drugs due to their 
aqueous core and lipid bilayer membrane. EVs has the advantage that they can be loaded with biological materials with high tolerability within the body due to inhibition of phagocytosis and complement system, with protection against chemicals and enzyme degradation. Furthermore, EVs are able to home to target tissue and deliver their cargo to recipient cells. Approaches involving viral packaging strategies, has been used to load therapeutic molecules into $\mathrm{EVs}^{[7]}$. Moreover, EVs were suggested as biomarkers for diagnostic and prognostic purpose ${ }^{[10]}$. Exosomes were detected in serum of patients with glioblastoma and ovarian cancer $^{[13]}$.

\section{Advantages of EVs over MSCs}

The employment of MSCs-derived EVs to replace MSC transplantation is of great interest as it could minimise the safety concerns regarding cell of origin and immunocompatibility ${ }^{[4]}$. EVs have enormous advantages over MSCs, including the ability to home to target tissue with no organ accumulation, no toxicity or long term maldifferentiation of engrafted cells. Moreover, no immune rejection or tumour generation following administration ${ }^{[7]}$ Additionally, EVs are able to deliver biological materials safer than methods based on liposome and viral vehicles. EVs are stable in different storage conditions, easily delivered with a half-life of 30-60 minutes and complete disappearance within 4 hours following intravenous administration $^{[34]}$.

\section{Challenges}

EVs isolation process includes ultracentrifugation, which is a costly and time consuming procedure requiring a large number of cells. Although faster reagents are available, still reported to precipitate contaminants of membrane fragments as lipoprotein ${ }^{[8]}$. A major challenge in EVs application is to identify a standardized, reliable and consistent method for isolation as the difference in isolation method gives rise to exosomes of different types and content, despite the use of the same source ${ }^{[1]}$.

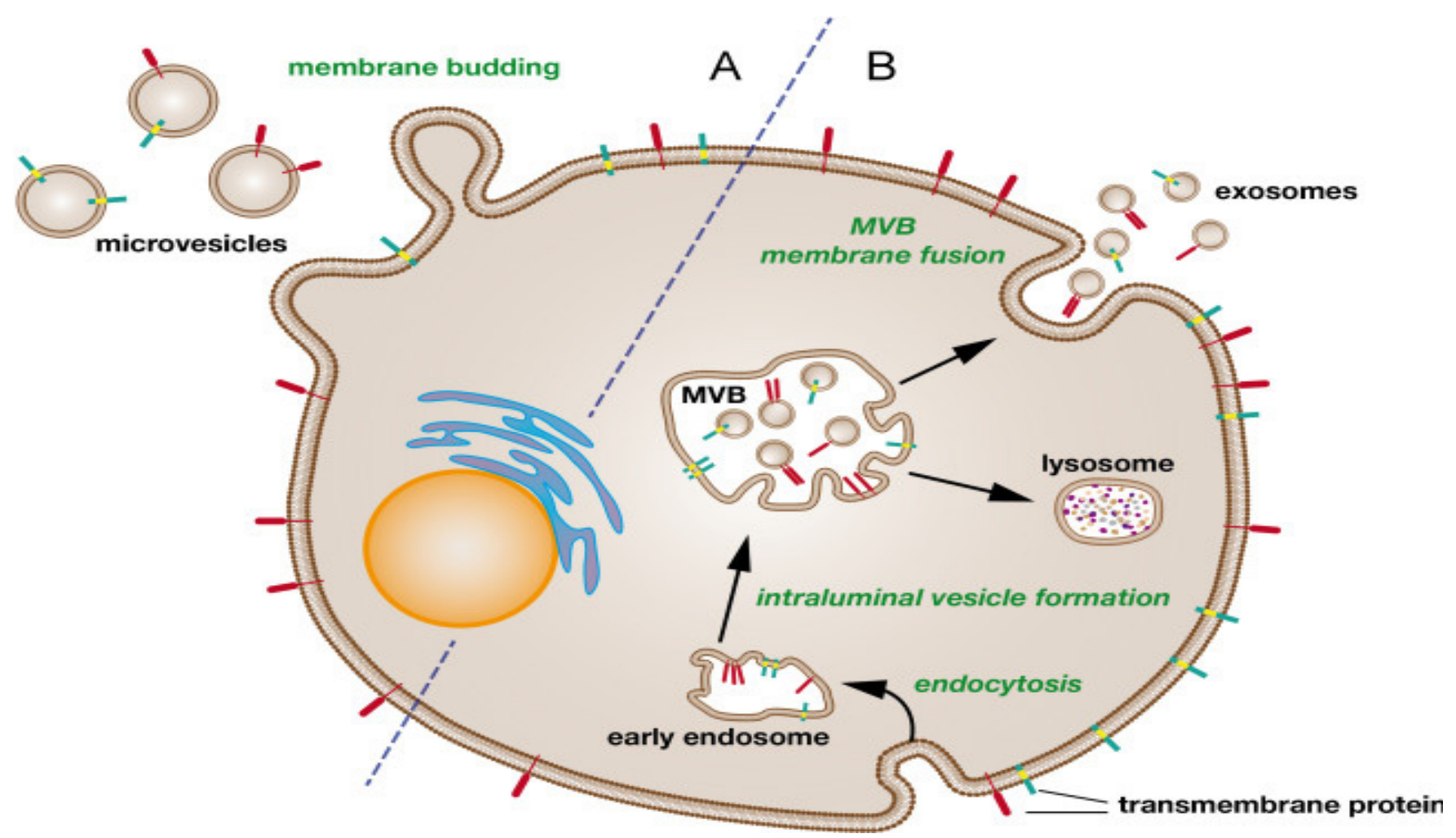

Fig. 1: Formation and release of extracellular vesicles (EVs). EVs are generating through different mechanisms. (A) Microvesicles are secreted from cells by budding directly from the plasma membrane; (B) exosomes are formed by inward budding of late endocytotic intraluminal vesicles called multivesicular bodies (MVBs). The MVB fuses with the plasma membrane to release the contained exosomes in the extracellular space.MVB $=$ multivesicular body ${ }^{[14]}$. 
MSCs-EVs. Abdel-Fattah, Lamyaa

Table 1: Characteristics of extracellular vesicles.

\begin{tabular}{|c|c|c|c|c|}
\hline Extracellular vesicles & Origin & Size & Content & Markers \\
\hline \multirow[t]{5}{*}{ Exosomes } & & & Proteins & CD9,CD63,CD81, \\
\hline & & & lipids & Hsp,Alix, Tsg101, \\
\hline & MVB & $50-150 \mathrm{~nm}$ & DNA & integrin, annexin, \\
\hline & & & mRNA & MHC I \& II \\
\hline & & & miRNA & \\
\hline \multirow[t]{7}{*}{ Microvesicles } & & & Proteins & Integrins \\
\hline & & & lipids & Flotillins \\
\hline & & & DNA & Tetraspanins \\
\hline & Plasma membrane & $150-1000 \mathrm{~nm}$ & & \\
\hline & & & mRNA & \\
\hline & & & miRNA & \\
\hline & & & Cell organelles & \\
\hline \multirow[t]{5}{*}{ Apoptotic bodies } & & & DNA & Surface markers \\
\hline & & & Noncoding- & of macrophage \\
\hline & Membrane $>1$ um of $d y$ & & & \\
\hline & & & RNAs & \\
\hline & & & Cell organelles & \\
\hline
\end{tabular}

Alix ALG-2-interacting protein X, MHC major histocompatibility complex, miRNA microRNA, Tsg101 tumour susceptibility gene 101, MVB multivesicular body (7).

\section{CONCLUSION}

Extracellular vesicles are promising biomedical tools in stem cell based therapies.Better understanding of mechanisms that control cargo loaded by EVs is extremely and interestingly needed. Many questions needed to be addressed including, which is the signal regulating the cargo of molecules within the EVs and which stimulus could trigger their release and which might supress their production, is there a reliable method to control signalling?. Moreover, the use of EVs alone or in addition to selected factors of the MSCs secretome would be more beneficial to reach the therapeutic goals efficiently. Many investigations are still needed to enable the use of EVs based therapy in clinical practice.

\section{CONFLICT OF INTEREST}

There are no conflicts of interest.

\section{REFERENCES}

1. Spees JL, Lee RH, Gregory CA. Mechanisms of mesenchymal stem/stromal cell function. Stem Cell Research and Therapy 2016; 7: 125- 137

2. Sharma RR, Pollock K, Hubel A, McKenna D.Mesenchymal stem or stromal cells: a review of clinical applications and manufacturing practices. Transfusion 2014;54 (5): 1418 -1437.

3. Skalnikova H, Motlik J, Gadher SJ ,Kovarova H. Mapping of the secretome of primary isolates of mammalian cells, stem cells and derived cell lines. Proteomics 2011; 11 (4): 691 -708.

4. Gallina C, TurinettoV, Giachino C. Anew paradigm in cardiac regeneration: The mesenchymal stem cell secretome. Stem cells International. 2015; 2015: 846- 855. ID 765846. 
5. Akyurekli C, Le Y, Richardson RB, Fergusson D, TayJ, Allan DS. A systematic review of preclinical studies on the therapeutic potential of mesenchymal stromal derived microvesicles. Stem Cell Rev. 2015; 11 (1): 150- 160.

6. Camussi G, Deregibus MC, Bruno S, Cantaluppi $\mathrm{V}$, Biancore L.Exosomes / microvesicles as a mechanism of cell-to-cell communication. Kidney Int. 2010;78(9): 838- 848 .

7. Abreu SC, Weiss DJ, Rocco PRM. Extracellular vesicles derived from mesenchymal stromal cells: a therapeutic option in respiratory diseases? Stem Cell Research and Therapy 2016;7: 53- 62.

8. De Jong O G, Van Balkom B W M, Schiffelers R M, Bouten C V C , Verharr M C. Extracellular vesicles : Potential roles in regenerative medicine. Front. Immunol. 2014; 5: $608-620$.

9. QuesenberryPJ, GoldbergLR, AliottaJMDo onerMS,Pereira MG, Wen S, Camussi G. cellular phenotype and extracellular vesicles: basic and clinical considerations. Stem Cells Dev. 2014; 23(13): 1429 -1436.

10. Biancone L, Bruno S, Deregibus MC, Tetta C, Camussi G. Therapeutic potential of mesenchymal stem cell-derived microvesicles. Nephrol. Dial. Transplant. 2012;27(8): 30373042.

11. Fujita Y, Kosaka N, Araya J, Kuwano K, Ochiya T. Extracellular vesicles in lung microenviroment and pathogenesis. Trends Mol. Med. 2015; 21(9): 533- 542.

12. Tomasoni S, Longaretti L, Rota C, Morigi M, Conti S, Gotti E, Capelli C, Introna M,Remuzzi $\mathrm{G}$, Benigni A. Transfer of growth factor receptor mRNA via exosomes unravels the regenerative effect of mesenchymal stem cells. Stem CellsDev. 2013; 22(5): 772- 780 .

13. EmanueliC, Shearn A I U, Angelini GD, Sahoo S. Exosomes and exosomalmiRNAs in cardiovascular protection and repair. Vascul. Pharmacol. 2015; 71:24- 30.

14. Junker K, Heinzelmann J, Beckham C, Ochiva T, Jenster G. Extracellular vesicles and their role in urologic malignancies. Europ. Urol. 2017; 70(2):323- 331.

15. Camussi G, Deregibus MC, Cantaluppi V. Role of stem cell-derived microvesicles in the paracrine action of stem cells. Biochemical Society
Transaction 2013; 41(1):283 -287.

16. Tetta C, Bruno S, Fonsato V, Deregibus MC, Camussi G. The role of microvesicles in tissue repair. Organogenesis 2011; 7 (2): 105- 115.

17. Chen J, Li C, Chen L. The role of microvesicles derived from mesenchymal stem cells in lung diseases. Biomed Research International. 2015 ; 2015: 814- 819.

18. Loyer X, Vion A-C, Tedgui A, Boulanger CM. Microvesicles as cell-cell messengers in cardiovascular diseases. Circulation Research 2014; 114: 345- 353.

19. Fierabracci A, Del Fattore A, Luciano R, Muraca M,TetiA.Recentadvancesinmesenchymalstemcell immunomodulation: the role of microvesicles. Cell Transplant. 2015; 24(2): 133- 149.

20. Zou X, Zhang G, Zhongliang C, Yin D, Du T, Ju G, Miao S, Liu G, Lu M, Zhu Y.Microvesicles derived from human Wharton's jelly mesenchymal stromal cells ameliorate renal ischaemia reperfusion injury in rats by suppressing CX3CL1. Stem Cell Research and Therapy 2014; 5: 40- 52.

21. Théry C, OstrowskiM, Segura E. Membrane vesicles as conveyors of immune responses. Nature reviews. Immunology. 2009; 9: 581-93.

22. Gardiner C, Ferreira YJ, Dragovic RA, Redman CW , Sargent IL. Extracellular vesicles sizing and enumeration by nanoparticle tracking analysis. J Extracell.Vesicles 2013;2: 19671- 19681.

23. Lopatina T, Bruno S, Tetta C, Kalinina N, Porta M, Camussi G. Platelet -derived growth factor regulates the secretion of extracellular vesicles by adipose mesenchymal stem cells and enhances their angiogenic potential. Cell Commun. Signal 2014: 12:26- 37.

24. Parolini I, Federici C, Raggi C, Lugini L, Palleschi S, De Milito A, Coscia C, Iessi E, Logozzi M, Molinari A, Colone M, Tatti M, Sargiacomo M ,Fais S.Microenviromental $\mathrm{PH}$ is a key factor for exosome traffic in tumour cells. $\mathrm{J}$ Biol. Chem. 2009; 284(49): 34211- 34222.

25. Cruz FFBZ, Goodwin M, Sokocevic D, Wagner DE, Coffey A, Antunes M, Robinson KL, Mitsials SA, Kourembanas S, Thane K, Hoffman AM, McKenna DH, Rocco PRM, Weiss DJ. Systemic administration of human bone marrowderived mesenchymal stromal cell extracellular vesicles ameliorates aspergillushyphal extract- 
induced allergic airway inflammation in immunocompetent mice. Stem Cell Transl Med. 2015;4(11): 1302-1316.

26. Lee C, Mitsialis SA, Aslam M, Vitali SH, Vergadi E, Konstantinou G, Sdrimas K, FernandezGonzalez A, Kourembanas S. Exosomes mediate the cytoprotective action of mesenchymal stromal cells on hypoxia-induced pulmonary hypertension. Circulation. 2012; 126(22): 2601-2611.

27. Lee JW, Fang X, Krasnodembskaya A, Howard JP, Matthay MA. Concise review: mesenchymal stem cells for acute lung injury: role of paracrine soluble factors. Stem Cells. 2011; 29(6): 913-919.

28. Monsel A, Zhu YG, Gennai S, Hao Q, Hu S, Rouby JJ, Rosenzwajg M, Matthay MA, Lee JW. Therapeutic effects of human mesenchymal stem cell-derived microvesicles in severe pneumonia in mice. Am J Respir Crit Care Med. 2015; 192(3): 324-336.

29. Wang $\mathrm{Y}$, Lu X, He J, Zhao W.Influence of erythropoietin on microvesicles derived from mesenchymal stem cells protecting renal function of chronic kidney disease. Stem Cell Res. and Therapy. 2015; 6 (1): 100- 113.
30. Zhang B, Yin Y, Lai RC, Tan SS, Choo AB, Lim SK.Mesenchymal stem cells secrete immunologically active exosomes. Stem Cells Dev. 2014; 23(11): 1233-1244.

31. Li X, Liu L, Yang J, Yu Y, Chai J, Wang L, Ma L, Yin H. Exosome Derived From Human Umbilical Cord Mesenchymal Stem Cell Mediates MiR181c Attenuating Burn-induced Excessive Inflammation. E Bio Medicine.2016; 8: 72- 82.

32. LeibacherJ ,Henchler R. Biodistribution, migration and homing of systemically applied mesenchymal stem/stromal cells. Stem Cell Research and Therapy 2016; 7: 7- 18 .

33. Lou G, Song X, Yang F, Wu S, Wang J, Chen $\mathrm{Z}$, Liu Y. Exosomes derived from miR-122modified adipose tissue-derived MSCs increase chemosensitivity of hepatocellular carcinoma. J Haematol.Oncol. 2015; 8: 122- 132.

34. Di Rocco G, BaldariS ,Toietta G. Towards Therapeutic Delivery of Extracellular Vesicles: Strategies for In Vivo Tracking and Biodistribution Analysis. Stem Cells Int. 2016; 2016: 619- 630. 